\title{
Diffusiophoresis in Cells: a General Non-Equilibrium, Non-Motor Mechanism for the Metabolism-Dependent Transport of Particles in Cells
}

\author{
Richard P. Sear \\ Department of Physics, University of Surrey, Guildford, GU2 7XH, UK*
}

\begin{abstract}
The more we learn about the cytoplasm of cells, the more we realise that the cytoplasm is not uniform but instead is highly inhomogeneous. In any inhomogeneous solution, there are concentration gradients, and particles move either up or down these gradients due to a mechanism called diffusiophoresis. I estimate that inside metabolically active cells, the dynamics of particles can be strongly accelerated by diffusiophoresis, provided that they are at least tens of nanometres across. The dynamics of smaller objects, such as single proteins are largely unaffected.
\end{abstract}

The cytoplasm of cells is far from thermodynamic equilibrium, and far from uniform [1-4]. Here, I consider the effect of concentration gradients on the motion of large particles in the cytoplasm. Large means tens of nanometres and above, so an example would be a large protein assembly. In the cytoplasm, particles and molecules are not diffusing alone in a dilute solution, but are moving in a concentrated, active and non-uniform mixture of proteins, nucleic acids, metabolites such as ATP, small ions such as potassium, etc. A schematic of a particle in the cytoplasm, is shown in Fig. 1.

It is well known in the fields of colloids [5-18] and of liquid mixtures $[10,19]$, that particles of one species will move in response to a gradient in the concentration of another species. In colloids this is called diffusiophoresis. Diffusiophoresis is typically defined $[5-8,10]$ as the motion of a larger particle immersed in concentration gradients of smaller molecules, when both are in a solvent (such as water). Although often difficult to measure, there are clearly gradients inside metabolically active cells. So there must be diffusiophoresis occurring in cells, the question is: Does diffusiophoresis make a significant contribution to the transport of some species? Here, I determine that the answer to this question is probably yes for particles at least tens of nanometres or more across, but no for individual protein molecules.

I start with the standard Brownian-dynamics approximation for the position of a particle, $\mathbf{r}(t)$ [20]. With this approximation, we can write the change in position over the time interval $t$ to $t+\delta t$, as [20],

$$
\begin{array}{r}
\mathbf{r}(t+\delta t)=\mathbf{r}(t)+\left(2 D_{\mathrm{P}}^{(c)} \delta t\right)^{1 / 2} \rho+ \\
{\left[\mathbf{v}_{\text {adv }}+\mathbf{U}\right] \delta t+\frac{D_{\mathrm{P}}^{(c)}}{k T} \mathbf{f} \delta t}
\end{array}
$$

This equation includes four possible transport mechanisms for the particle. The second term on the right-hand side is the conventional thermal diffusion term. There $D_{\mathrm{P}}^{(c)}$ is the diffusion constant for thermal diffusion in the cytoplasm, and $\rho$ is a vector of random numbers drawn from a Gaussian distribution of mean zero, and standard deviation one. The physics of this term is that the particle is constantly being bombarded by the surrounding molecules, due to their thermal energy. This tends to move the particle around, but this motion is opposed by the friction between a moving particle and these same molecules.

The third term on the right-hand side contains the advection and phoresis terms. Advection is motion of a particle because it is carried along by the cytoplasm flowing at a local velocity $\mathbf{v}_{\text {adv }}$. $\mathbf{U}$ is the diffusiophoretic velocity. If the cytoplasm is inhomogeneous (has gradients) at a point, then locally the stresses on the particle are also inhomogeneous, which means that there are unbalanced stresses which will cause the particle to move relative to the local fluid [5, 6, 8, 12-14]. This local motion is called a slip velocity, and can be caused by gradients in anything. Here we will consider gradients in concentration, and then this slip velocity is a diffusiophoretic velocity, $\mathbf{U}$. Both $\mathbf{U}$ and $\mathbf{v}_{\text {adv }}$ are zero in a system at equilibrium, so in a cell they must come from the cell's metabolism.

The last term on the right is motion due to a force f on the particle, for example due to a motor protein pushing or pulling on the particle. In eukaryote cells, it is well established that motor proteins pull many cargos around the cell. Although this is an important process, it is well studied [21] and so here I only consider particles not being pulled by motor proteins.

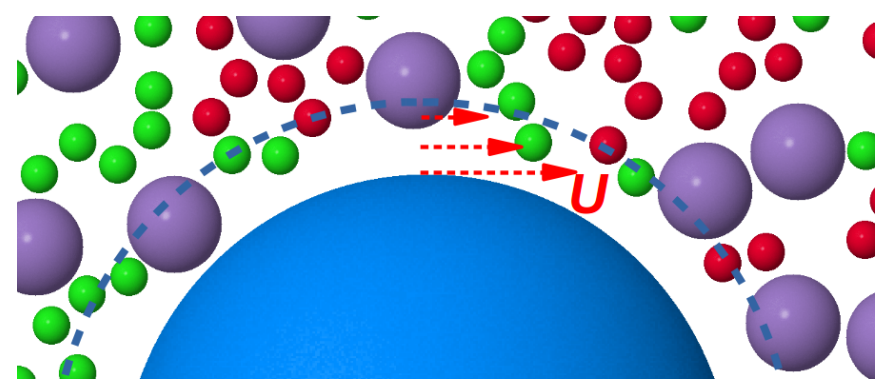

FIG. 1. Schematic of a particle (blue), immersed in a cytoplasm with gradients in the concentrations of two metabolites (red and green). Proteins are magenta. Small ions are not shown. The dotted line indicates the approximate extent of the particle/cytoplasm interface, where slip occurs, creating a gradient in velocity, and hence the diffusiophoretic slip velocity $\mathbf{U}$. 
To motivate this study, let us consider experimental evidence for metabolism-dependent mobility of particles in cells. Parry et al. [22] studied the dynamics of large, around 50 to $150 \mathrm{~nm}$ across, particles in the cytoplasm of bacteria (including $E$. coli). The particles included granules of an enzyme, a plasmid (of a type without an active partitioning system), and particles formed by a self-assembling viral protein. They found that the dynamics of particles in this size range, dramatically slowed down when the metabolism was shut off. The metabolism was shut off by depleting ATP and GTP using 2,4-dinitrophenol (DNP).

Parry et al. [22] tracked the displacement of particles $\sim 100 \mathrm{~nm}$ across over periods of $15 \mathrm{~s}$. When the metabolism was shut down, the particles made many fewer displacements of order hundreds of nanometres, and this dramatically slowed movement. So we are looking for a metabolism-dependent mechanism that can transport assemblies $100 \mathrm{~nm}$ across at an effective speed of up to $\sim 100 \mathrm{~nm} / \mathrm{s}$ for periods of $10 \mathrm{~s}$. Here I suggest that diffusiophoresis is a possible mechanism.

It is worth noting that both with and without an active metabolism, the distribution of displacements was very far from the Gaussian distribution expected for thermal diffusion in a uniform background. This non-Gaussian distribution implies that the cytoplasm is strongly nonuniform.

The results of Parry et al. [22] are for bacteria. The presence of motors and the cytoskeleton in eukaryote cells, will make it difficult to unambiguously observe diffusiophoresis in eukaryotes. However, I note that Bajanca et al. [23] studied the motion of the protein dystrophin in the muscle cells of zebrafish embryos. This protein has been estimated to be $100 \mathrm{~nm}$ long. They found effective diffusion constants of order $1 \mu \mathrm{m}^{2} / \mathrm{s}$, only an order of magnitude lower than that of GFP $(\sim 3 \mathrm{~nm}$ across) in the same cells. The effective diffusion constant of dystrophin is seems too large to be consistent with the Stokes-Einstein expression for thermal diffusion, assuming an effective cytoplasmic viscosity ten times that of water. A cytoplasmic viscosity ten times that of water is consistent with the measured diffusion constant for GFP [24]. This leaves us looking for a transport mechanism beyond simple thermal diffusion.

I am not the first to consider phoretic motion in cells, Lipchinsky [25] considered osmophoresis, motion driven by a gradient in the osmotic pressure, in pollen tubes. As the osmotic pressure gradient is due to a gradient in the concentration of small ions, osmophoresis is a type of diffusiophoresis. Ietswaart et al. [26], Surovtsev et al. [27], and Walter et al. [28] all modelled what is called the ParA/B [29] system of segregating plasmid DNA in bacteria during cell division. The plasmid moves in a concentration gradient of the ParA protein, and so their work [26-28] is an example of diffusiophoresis. However, the molecular interactions and stresses responsible for the plasmid motion were not explicitly modelled in that work $[26,28]$. Here I do consider these interactions and stresses here, and so my work is complementary to that earlier work [26, 28]. Surovtsev et al. [27] used a Brownian dynamics model for the interaction, this may overestimate the strength of diffusiophoresis, as discussed by Sear and Warren [8, 9].

There are thousands of species inside cells, many of which may have gradients. To keep things simple, I work with the gradient in just one example species: the abundant metabolite ATP. I select ATP as a test candidate as it is known to interact strongly with proteins at the concentrations found in cells [30], and to turnover rapidly [21]. The rapid turnover implies large fluxes between the sources and sinks, and the fluxes imply gradients, between these sources and sinks. Thus ATP is my best candidate for an abundant species whose concentration gradients I can estimate. When ATP is consumed ADP is produced, so although here I will refer to an ATP gradient for simplicity, in reality it is two gradients, one of ATP and one of ADP, with the opposite sense. The effects of these two opposing gradients may partially cancel, weakening diffusiophoresis, but as the molecules are different, any cancellation will be partial. Note that small ions such as potassium and chloride are even more abundant than ATP inside cells, but as they do not turnover are expected to have only negligible concentration gradients. The numbers needed to characterise cells in my calculations are gathered together in Table I in the Supplemental Material. A particle moving up an ATP gradient is shown in Fig. 2.

Inside cells, thermal energy and momentum can move much more rapidly than even small molecules. So, I expect thermal and pressure gradients to be negligible, see the Supplemental Material for the justification of this assumption.

In order to estimate the sizes of the gradients in ATP concentration inside cells, I start by estimating the timescale for ATP to diffuse across a typical bacterial cell $1 \mu \mathrm{m}$ across. The diffusion constant of ATP both in water and in cells $[21,31]$ is of order $100 \mu \mathrm{m}^{2} / \mathrm{s}$. So an ATP molecule diffuses across the cell in of order $0.01 \mathrm{~s}$.

An active $1 \mu \mathrm{m}^{3}$ bacterial cell is estimated to have $10^{7}$ ATP molecules and to consume $10^{7}$ ATP molecules each second, see Table I of the Supplemental Material. This gives a time of $1 \mathrm{~s}$ between production by ATP synthase, and consumption. A lifetime 100 times the diffusion time implies gradients of order $1 \%$ to $10 \%$ across a cell $1 \mu \mathrm{m}$ across. For an ATP concentration of $10^{7} / \mu^{3}$, we have gradients of $10^{5} / \mu \mathrm{m}^{4}$ to $10^{6} / \mu \mathrm{m}^{4}$. I will use the gradient value $10^{5} / \mu \mathrm{m}^{4}$ below. See the Supplemental Material for a more detailed calculation that also gives gradients of this size. These are very simple estimates of steady-state gradients, the gradient will presumably vary in space and time as particular sources (ATP synthase) and sinks (ATP consuming proteins) move. But as 
ATP diffuses much faster than membrane proteins such as ATP synthase, ATP gradients may often be close to a steady state.

The diffusiophoretic velocity $\mathbf{U}$ is proportional to the gradient in the concentration $c$, of a solute

$$
\mathbf{U}=\Lambda_{\mathrm{PH}} \nabla c
$$

There is a standard Derjaguin/Anderson expression [6, $8,12,32]$ for the coefficient $\Lambda_{\mathrm{PH}}$ that relates the concentration gradient to the diffusiophoretic velocity. This expression is valid for a large particle with an interaction $\phi(z)$ between the particle surface and a smaller species that has a concentration gradient $\nabla c$. Here $z$ is the distance separating the smaller species from the surface of the particle. Between the smaller species and the surface is a continuum solvent with viscosity $\eta$. The Derjaguin/Anderson expression is

$$
\Lambda_{\mathrm{PH}}=\frac{k_{\mathrm{B}} T}{\eta} \int_{0}^{\infty} z\left[\exp \left(-\phi(z) / k_{\mathrm{B}} T\right)-1\right] \mathrm{d} z
$$

Note that as the particle surface is interacting with the smaller species in water, $\phi(z)$ is an effective interaction free energy.

From Eq. (3), we see that the diffusiophoretic coefficient $\Lambda_{\mathrm{PH}}$ is approximately $k_{\mathrm{B}} T$ divided by the solvent viscosity $\eta$, and multiplied by the square of the interaction range, which we denote by $L$. So, we obtain the approximate expression

$$
\Lambda_{\mathrm{PH}} \sim \pm k_{\mathrm{B}} T L^{2} / \eta
$$

$\Lambda_{\mathrm{PH}}$ is positive for attractive interactions, and then $\mathbf{U}$ is directed to higher concentrations of the solute. For repulsive interactions the sign is reversed. The integral in Eq. (3) is of order $-L^{2}$ for a repulsive $\phi(z)$ that is

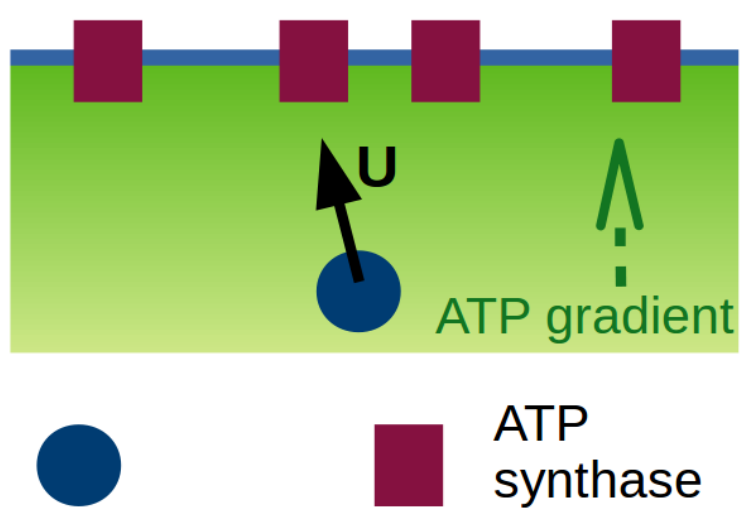

FIG. 2. Schematic of part of a prokaryote cell, with an ATP concentration gradient indicated by shading. Sources of the gradient are ATP synthases, in magenta, while our model assumes that sinks (ATP consuming proteins) are uniformly distributed in the cytoplasm. We show one particle moving up the concentration at a diffusiophoretic velocity $\mathbf{U}$. $\sim k_{\mathrm{B}} T$ or stronger over a range $L$, and is of order $+L^{2}$ for an attractive $\phi(z)$ that is of order $k_{\mathrm{B}} T$ over a range $L$. For a stronger attraction, the integral will be larger, but Eq. (3) is an approximation [6, 8, 12, 32], and will break down for strong enough attractions. To summarise, the approximation of Eq. (4) should be the correct order of magnitude unless there are attractions $\gg k_{\mathrm{B}} T$ in which case the Derjaguin/Anderson approximation fails. So I do need to assume that, for the particles studied by Parry et al. [22], the interactions between the protein and ATP are not strongly $\left(\gg k_{\mathrm{B}} T\right)$ attractive.

Here we estimate the diffusiophoretic velocity $\mathbf{U}$ of a particle in a concentration gradient of ATP. The diffusiophoretic coefficient depends on the free energy of particle/ATP interaction $\epsilon$, the range of the surface/ATP interaction $L$, and the solvent viscosity $\eta$. I approximate the viscosity by that of water, $\eta \sim 10^{-3} \mathrm{~Pa}$ s. The free energy of interaction $\epsilon$, I take to be $k_{\mathrm{B}} T=4 \times 10^{-21} \mathrm{~J}$, and the range $L$ to be $1 \mathrm{~nm}$. From ATP's diffusion coefficient of $500 \mu \mathrm{m}^{2} / \mathrm{s}$, ATP has a Stokes-Einstein radius of $0.7 \mathrm{~nm}$. Then $\Lambda_{\mathrm{PH}}=4 \times 10^{-18} \mu \mathrm{m}^{3} / \mathrm{s}$, and

$$
U \sim 4 \times 10^{-18}\left|\nabla c_{\mathrm{ATP}}\right| \quad\left[\nabla c_{\mathrm{ATP}} \text { in } \mu \mathrm{m}^{-4}\right]
$$

for $c_{\text {ATP }}$ the ATP concentration. We set $L=1 \mathrm{~nm}$, as that is the order of magnitude of both the size of ATP itself and of the Debye screening length in the cytoplasm. ATP is both highly charged and contains organic groups, so its nature is a little amphiphilic. Therefore, the interactions with a protein surface will be complex [30] but will include electrostatic interactions, with a range of the Debye length. Interactions beyond a few nanometres are expected to be weak [33].

Above, we estimated the gradient in ATP concentration to be $10^{5} / \mu \mathrm{m}^{4}=10^{29} / \mathrm{m}^{4}$. Putting that gradient in Eq. (5), we have a diffusiophoretic speed $U \sim 400 \mathrm{~nm} / \mathrm{s}$. This is large enough to be consistent with the motion observed by Parry et al. [22], so long as the gradient lasts for of order $10 \mathrm{~s}$ or more. Our estimates for the gradients, are steady-state estimates, so they should satisfy this constraint.

This is the key result of this work: Physically reasonable concentration gradients of one abundant metabolite, can drive motion of large particles that is fast enough to be significant for transport inside cells, and fast enough to be observable. Note that as typical proteins diffuse across a $1 \mu \mathrm{m}$ cell in less than 1 second, an additional speed of $100 \mathrm{~nm} / \mathrm{s}$ has little effect on the dynamics of single proteins, so diffusiophoresis should not affect significantly affect protein dynamics.

My estimate of speeds of hundreds of nanometres per second is highly approximate, so I would like to comment on sources of uncertainty. It relies on my estimate of the gradients. These could be out by an order of magnitude, and it is difficult to assess how gradients vary in space and time. It is also worth noting if the phoretic velocity is directed towards a source of a gradient, there will 
be positive feedback as particles will be pulled towards the source where the gradient is steepest, an effect that is magnified when the source itself can move [34]. The phoretic interaction could pull the particle into contact with the source, where the concentration gradients are strongest. This was a theory and simulation study. Experiments in vitro by Zhao et al. [35] found that phoretic interactions can help enzymes move together. Thus our estimates for $U$ may be underestimates when the phoretic velocity is towards gradient sources.

The estimated speed also relies on our value for $\Lambda_{\mathrm{PH}}$. The Anderson-Derjaguin expression [6, 8, 12, 32] applies to dilute systems (the cytoplasm is not dilute), and relies on flow in a fluid interfacial region of width $L$, driven by the stresses there. It is uncertain how good these approximations are in the cytoplasm.

There have been (in vitro) experimental studies of proteins moving due to active processes. Sen and coworkers [35-38], and Granick and coworkers [39], have both studied enzymes, such as urease, in dilute solution. Both groups find that enzymes move faster when they are catalysing reactions, and Zhao et al. [36] also found that active enzymes could speed up the motion of other species. Future work could consider solutions with concentrations of energy-consuming molecules that are closer to those found in the cytoplasm. Jee et al. [39] have already considered the effect of a crowding agent. Future work could also use microfluidics to create gradients in ATP, in order to look for phoresis.

My estimate is for prokayotes. Milo et al. [21] discuss the energy consumption of mammalian cells. The power consumption per unit volume of a fibroblast can be comparable to that of E. coli. Assuming distances of a few micrometres between where ATP is consumed, and mitochondria, the ATP gradients in an active fibroblast will be comparable to those in growing E. coli. So diffusiophoretic speeds should also be comparable.

We have only considered a gradient in one of the thousands of species in a cell (ATP), and models of the ParA/B system of moving plasmids in bacteria [27-29] also only consider ParA gradients. Future work will need to deal with the multicomponent nature of the cytoplasm. Systems that have evolved to localise species such as plasmids presumably have to work against the forces due to flutuating gradients in the other species present in the cell.

Diffusiophoresis is unlikely to be the only non-motordriven metabolism-dependent transport mechanism in cells. See the Supplemental Material for more discussion of these other potential transport mechanisms. In eukaryote cells, there is also transport of particles as the cargos of motor proteins.

In conclusion, the more we learn of the cytoplasm of both prokaryote and eukaryote cells, the less uniform they appear to us [1-4]. There must be many gradients in cells, and so phoresis must be occurring in essentially all cells. However, quantifying phoretic speeds in cells is difficult. Cells are complex, and the size of gradients is unknown. In addition the interactions needed to estimate diffusiophoretic coefficients $\Lambda_{\mathrm{PH}}$ are also unknown. Here I estimated $\Lambda_{\mathrm{PH}}$ for ATP, and estimated the size of gradients of ATP in an active bacterial cell such as E. coli. I predicted that diffusiophoretic speeds of order $100 \mathrm{~nm} / \mathrm{s}$ are possible. This is large enough to be consistent with the motions observed by Parry et al. [22], for large (50 to $150 \mathrm{~nm}$ ) particles. However, the complexity of the cytoplasm means that is very difficult to unambiguously show that observed movements are due to one specific transport mechanism. Experiments on simpler, in vitro, systems will probably be required to separate out different non-thermal-diffusion contributions to transport in cells.

I would like to thank Patrick Warren for teaching me much of what I know about diffusiophoresis, and Daan Frenkel for many illuminating discussions. I would also like to thank the organisers, Julian Shillcock, Mikko Haataja and John Ipsen, and participants of the CECAM workshop Liquid Liquid Phase Separation in Cells, for helpful questions and feedback. The author confirms that no new data were created during this study.

* r.sear@surrey.ac.uk; https://richardsear.me/

[1] K. Luby-Phelps, in Microcompartmentation and Phase Separation in Cytoplasm, Int. Rev. Cyt., Vol. 192, edited by H. Walter, D. E. Brooks, and P. A. Srere (Academic Press, 1999) pp. $189-221$.

[2] P. S. Agutter and D. N. Wheatley, Bioessays 22, 1018 (2000).

[3] Y. Shin and C. P. Brangwynne, Science 357, 1253 (2017).

[4] J. B. Woodruff, B. F. Gomes, P. O. Widlund, J. Mahamid, A. Honigmann, and A. A. Hyman, Cell 169, 1066 (2017).

[5] J. L. Anderson, Ann. New York Acad. Sci. 469, 166 (1986)

[6] J. L. Anderson, Ann. Rev. Fluid Mech. 21, 61 (1989).

[7] E. Ruckenstein, J. Coll. Int. Sci. 83, 77 (1981).

[8] J. F. Brady, J. Fluid Mech. 667, 216 (2011).

[9] R. P. Sear and P. B. Warren, Phys. Rev. E 96, 062602 (2017).

[10] J. S. Paustian, C. D. Angulo, R. Nery-Azevedo, N. Shi, A. I. Abdel-Fattah, and T. M. Squires, Langmuir 31, 4402 (2015).

[11] S. Shin, E. Um, B. Sabass, J. T. Ault, M. Rahimi, P. B. Warren, and H. A. Stone, Proc. Nat. Acad. Sci. USA 113, 257 (2016).

[12] S. Marbach, H. Yoshida, and L. Bocquet, J. Chem. Phys. 146, 194701 (2017).

[13] H. Yoshida, S. Marbach, and L. Bocquet, J. Chem. Phys. 146, 194702 (2017).

[14] L. Bocquet and E. Charlaix, Chem. Soc. Rev. 39, 1073 (2010).

[15] D. Florea, S. Musa, J. M. Huyghe, and H. M. Wyss, Proc. Natl. Acad. Sci. USA 111, 6554 (2014). 
[16] D. Velegol, A. Garg, R. Guha, A. Kara, and M. Kumara, Soft Matter 12, 4686 (2016).

[17] S. Shin, P. B. Warren, and H. A. Stone, Phys. Rev. Applied 9, 034012 (2018).

[18] D. C. Prieve, S. M. Malone, A. S. Khair, R. F. Stout, and M. Y. Kanj, Proc. Nat. Acad. Sci. (2018).

[19] M. S. McAfee and O. Annunziata, Fluid Phase Equilibria 356, 46 (2013).

[20] M. P. Allen and D. J. Tildesley, Computer Simulation of Liquids, 2nd ed. (Clarendon Press, Oxford, 2017).

[21] R. Milo and R. Phillips, Cell Biology by the Numbers (Garland Science, 2015).

[22] B. R. Parry, I. V. Surovtsev, M. T. Cabeen, C. S. O'Hern, E. Dufresne, and C. Jacobs-Wagner, Cell 156, 183 (2014).

[23] F. Bajanca, V. Gonzalez-Perez, C. B. Sean J Gillespie, L. Garcia, E. Theveneau, R. P. Sear, and S. M. Hughes, eLife 4, e06541 (2015).

[24] P. Montero Llopis, O. Sliusarenko, J. Heinritz, and C. Jacobs-Wagner, Biophys. J. 103, 1848 (2012).

[25] A. Lipchinsky, Phys. Bio. 12, 066012 (2015).

[26] R. Ietswaart, F. Szardenings, K. Gerdes, and M. Howard, PLOS Comp. Biol. 10, 1 (2014).

[27] I. V. Surovtsev, M. Campos, and C. Jacobs-Wagner, Proc. Nat. Acad. Sci. 113, E7268 (2016).

[28] J.-C. Walter, J. Dorignac, V. Lorman, J. Rech, J.-Y.
Bouet, M. Nollmann, J. Palmeri, A. Parmeggiani, and F. Geniet, Phys. Rev. Lett. 119, 028101 (2017).

[29] I. V. Surovtsev and C. Jacobs-Wagner, Cell 172, 1271 (2018).

[30] A. Patel, L. Malinovska, S. Saha, J. Wang, S. Alberti, Y. Krishnan, and A. A. Hyman, Science 356, 753 (2017).

[31] R. A. de Graaf, A. van Kranenburg, and K. Nicolay, Biophys. J. 78, 1657 (2000).

[32] J. L. Anderson, M. E. Lowell, and D. C. Prieve, J. Fluid Mech. 117, 107 (1982).

[33] J. N. Israelachvili, Intermolecular and Surface Forces, 3rd ed. (Academic Press, 2011).

[34] S. Y. Reigh, P. Chuphal, S. Thakur, and R. Kapral, Soft Matter 14, 6043 (2018).

[35] X. Zhao, H. Palacci, V. Yadav, M. M. Spiering, M. K. Gilson, P. J. Butler, H. Hess, S. J. Benkovic, and A. Sen, Nature Chem. 10, 311 (2018).

[36] X. Zhao, K. K. Dey, S. Jeganathan, P. J. Butler, U. M. Crdova-Figueroa, and A. Sen, Nano Letters 17, 4807 (2017).

[37] X. Zhao, K. Gentile, F. Mohajerani, and A. Sen, Accounts of Chemical Research 51, 2373 (2018).

[38] F. Mohajerani, X. Zhao, A. Somasundar, D. Velegol, and A. Sen, Biochemistry 57, 6256 (2018).

[39] A.-Y. Jee, S. Dutta, Y.-K. Cho, T. Tlusty, and S. Granick, Proc. Nat. Acad. Sci. 115, 14 (2018). 